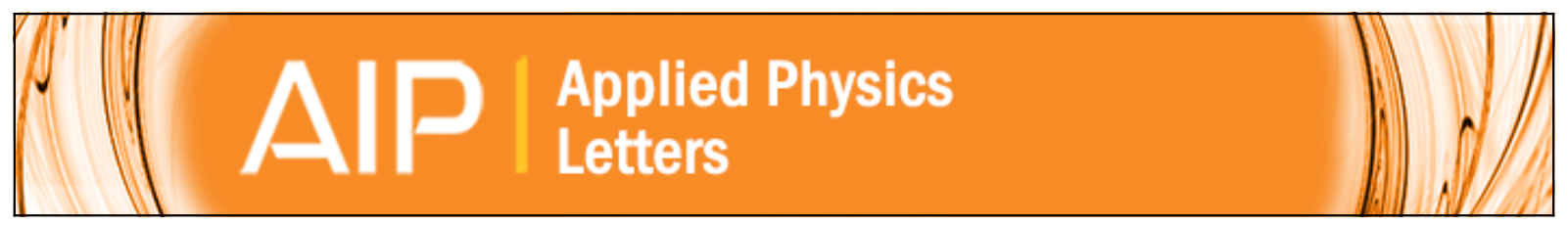

Large anomalous enhancement of perpendicular exchange bias by introduction of a nonmagnetic spacer between the ferromagnetic and antiferromagnetic layers

F. Garcia, J. Sort, B. Rodmacq, S. Auffret, and B. Dieny

Citation: Applied Physics Letters 83, 3537 (2003); doi: 10.1063/1.1619562

View online: http://dx.doi.org/10.1063/1.1619562

View Table of Contents: http://scitation.aip.org/content/aip/journal/apl/83/17?ver=pdfcov

Published by the AIP Publishing

\title{
AplP $\operatorname{Re}$-register for Table of Content Alerts
}

\section{Create a profile.}




\title{
Large anomalous enhancement of perpendicular exchange bias by introduction of a nonmagnetic spacer between the ferromagnetic and antiferromagnetic layers
}

\author{
F. Garcia, J. Sort, ${ }^{\text {a) }}$ B. Rodmacq, S. Auffret, and B. Dieny \\ SPINTEC, CEA/Grenoble, 38054 Grenoble Cedex 9, France
}

(Received 14 March 2003; accepted 23 August 2003)

In $(\mathrm{Pt} / \mathrm{Co})_{n} / \mathrm{FeMn}$ multilayers, the magnitude of exchange bias, $H_{E}$, can be considerably enhanced by placing an ultrathin nonmagnetic Pt spacer between the multilayer (ML) and the antiferromagnetic (AFM) layer. The bias is maximum for a spacer layer thickness, $t$, of a few angstroms and it decreases progressively as $t$ is further increased. This bias enhancement is accompanied by an increase of coercivity, $H_{C}$. This behavior is due to the role of the Pt spacer in enhancing the perpendicular effective anisotropy of the last Co layer in the ML, which has the effect of increasing the net ferromagnetic (FM)/AFM spin projection, thus leading to the $H_{E}$ and $H_{C}$ enhancements. The decrease of $H_{E}$ and $H_{C}$ for thicker spacer layers is due to the limited range of the FM-AFM proximity effect. (C) 2003 American Institute of Physics. [DOI: 10.1063/1.1619562]

During the last decades, much attention has been focused on exchange-coupled ferromagnetic (FM)antiferromagnetic (AFM) bilayers, because of both their underlying physics and their applications. ${ }^{1,2}$ These materials exhibit a shift of the hysteresis loop (exchange bias, $H_{E}$ ) and an enhanced coercivity, $H_{C}$, when they are field cooled through the blocking temperature of the AFM. In spite of extensive research, the physical mechanisms governing exchange bias still remain controversial. ${ }^{3}$ However, the majority of models elaborated so far consider that exchange bias mainly originates from a short-range FM-AFM interaction. Only a few studies have shown the existence of exchange bias across a nonmagnetic spacer located between the FM and the AFM layers. ${ }^{4}$ Nevertheless, up to now, these studies have always reported an exponential decrease of $H_{E}$ as a function of the spacer thickness. ${ }^{4}$ In this letter we demonstrate that by depositing a Pt spacer layer between a FM multilayer (ML) and an AFM it is possible to increase the magnitude of $H_{E}$.

It is noteworthy that $H_{E}$ has been recently, to some extent, increased by diluting the AFM by nonmagnetic defects located in its bulk. ${ }^{5}$ Similar $H_{E}$ enhancements have been obtained by ion irradiating, in a controlled way, FM-AFM bilayers. ${ }^{6}$ All these results have been interpreted taking into account the role of impurities or defects as pinning centers for domain walls motion in the volume part of the AFM, ${ }^{5-7}$ or considering local stress-induced increase of the AFM anisotropy. ${ }^{8}$ Nevertheless, at the FM-AFM interface, nonmagnetic impurities are usually assumed to reduce the exchange coupling constant, $J_{\text {INT }}$, and consequently also $H_{E} \cdot{ }^{4-8}$

Furthermore, although in the past most exchange bias investigations have been carried out in thin films with inplane easy axis, it has been recently shown that it is also possible to induce exchange bias in multilayers exhibiting a perpendicular-to-film uniaxial anisotropy, e.g., Pt/Co ML. ${ }^{9-13}$ These multilayered structures are very appealing from both fundamental and applied points of view. For in-

${ }^{a)}$ Electronic mail: sort@ drfmc.ceng.cea.fr stance, the FM uniaxial anisotropy in these systems (and also $H_{C}$ and the saturation magnetization, $M_{S}$ ) can easily be tuned by simply varying the number of ML repeats or the relative thickness of the $\mathrm{Pt}$ and Co layers. ${ }^{11,12}$ Perpendicular exchange bias also offers the possibility to prepare spin valves or tunnel junctions with perpendicular-to-plane magnetization $^{14,15}$

Different series of multilayers with the composition $[\operatorname{Pt}(20 \AA) / \operatorname{Co}(4 \AA)]_{n} / \mathrm{Pt}(t) / \mathrm{FeMn}(130 \AA)$, with $n=2$, 3 , and 5 and $t$ between 0 and $20 \AA$, were deposited onto thermally oxidized $\mathrm{Si}$ wafers by dc magnetron sputtering and capped with a $20 \AA$ Pt layer. Some other samples (described in the following paragraphs) were also prepared. The samples were cooled from $150{ }^{\circ} \mathrm{C}$ (i.e., above the blocking temperature) under a field of $2.5 \mathrm{kOe}$, applied perpendicular to the film plane. Structural characterization was performed by $\mathrm{x}$-ray diffraction (XRD) and high-resolution transmission electron microscopy (HRTEM), while hysteresis loops were measured, perpendicular to the thin film direction, using the extraordinary Hall effect (EHE). ${ }^{16}$

HRTEM images, together with XRD patterns, reveal that both the (Pt/Co) ML and FeMn are polycrystalline with a weak (111) texture. No significant differences in the microstructure were observed between samples with or without the Pt spacer at the FM-AFM interface.

Typical hysteresis loops of the $(\mathrm{Pt} / \mathrm{Co})_{n} / \mathrm{Pt}(t) / \mathrm{FeMn}$ systems for $n=2$ and 5, measured at room temperature, are shown in Figs. 1(a) and 1(b), respectively. The loops correspond to $t=0,2,4$, and $20 \AA$. The $(\mathrm{Pt} / \mathrm{Co})_{n} / \mathrm{FeMn}$ system exhibits a crossover from in-plane to perpendicular-to-plane easy axis when increasing the number of $(\mathrm{Pt} / \mathrm{Co})$ repeats, ${ }^{11,15}$ due to the interplay between the perpendicular anisotropy induced by the $\mathrm{Co} / \mathrm{Pt}$ interfaces and the large in-plane anisotropy induced at the $\mathrm{Co} / \mathrm{FeMn}$ interface together with the shape anisotropy. A similar effect is observed for low number of repeats as the Pt spacer thickness increases. As can be seen in Fig. 1(a), for $n=2$ the loops become more square as $t$ increases. A similar trend is observed for $n=3$. However, for $n=5$ all the loops already exhibit a square shape, due to a strong perpendicular anisotropy. 

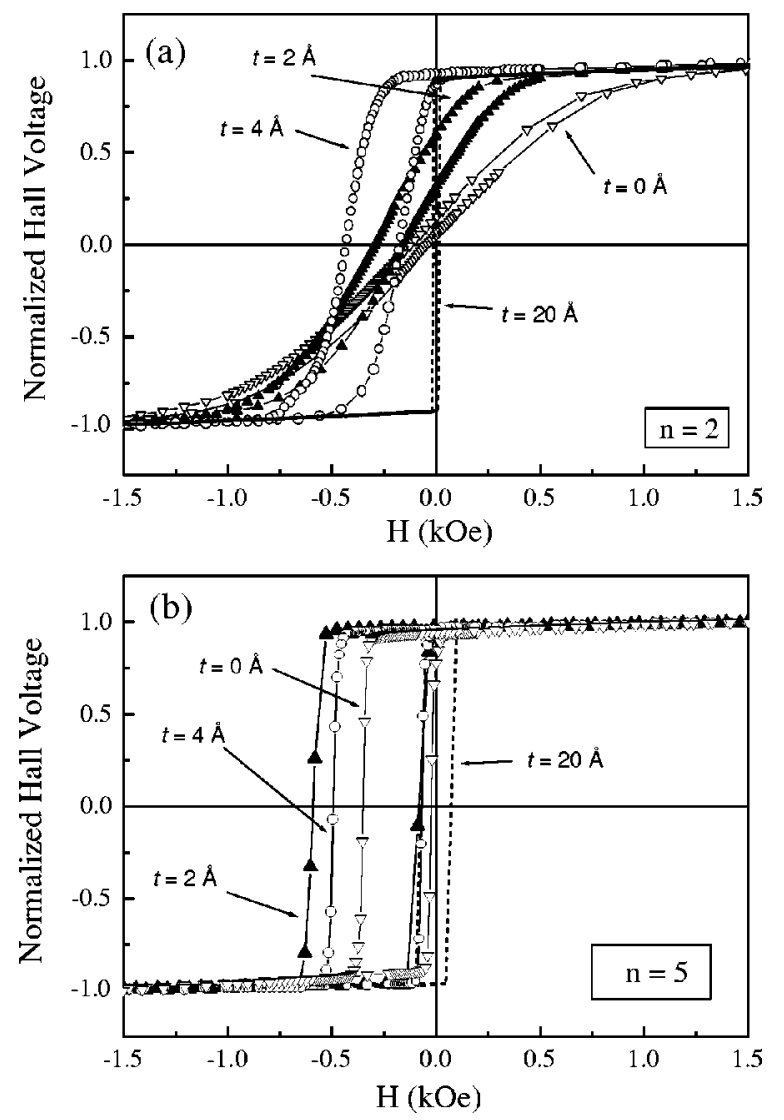

FIG. 1. Normalized hysteresis loops corresponding to $[\mathrm{Pt}(20 \AA) / \mathrm{Co}(4 \AA)]_{n} / \mathrm{Pt}(t) / \mathrm{FeMn}(130 \AA)(t=0,2,4$, and $20 \AA)$, for (a) $n=2$ and (b) $n=5$.

The dependence of $H_{E}$ on the Pt spacer layer thickness, $t$, for $n=2,3$, and 5 is shown in Fig. 2. It can be seen that $H_{E}$ increases significantly for low values of $t$, reaching a maximum at $t=5,2$, and $1 \AA$, respectively. When $t$ is further increased, $H_{E}$ progressively decreases. Moreover, the $H_{E}$ enhancement is more pronounced for lower values of $n$ (for $n$ $=2$, the bias increases by a factor of 4 , whereas for $n=5$ the enhancement is of about a factor of 2). The increase of $H_{E}$ is accompanied by an enhancement of $H_{C}$. This can be seen in the inset of Fig. 2, where the dependence of $H_{C}$ on $t$ is plotted for $n=2,3$, and 5. The increase of $H_{C}$ vs $n$ can be ascribed to the increase of the ML perpendicular anisotropy.

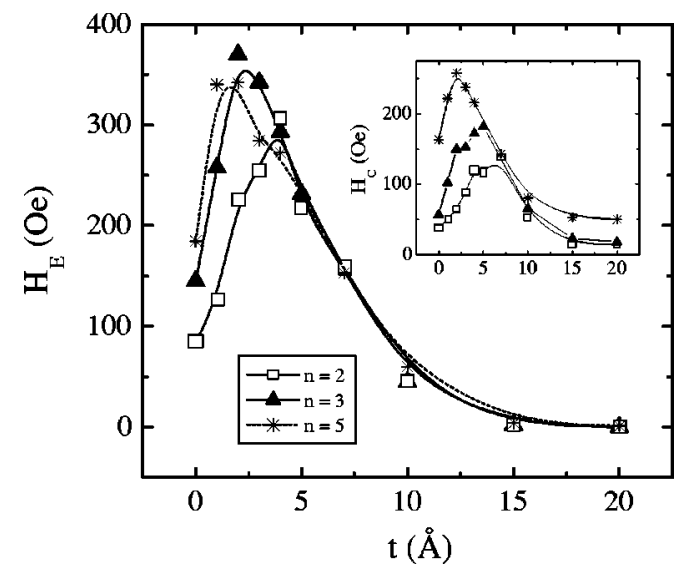

FIG. 2 Dependence of the exchange bias field, $H_{E}$, and the coercivity $H_{C}$ (in the inset), on the Pt spacer thickness, $t$, for $n=2,3$, and 5 .

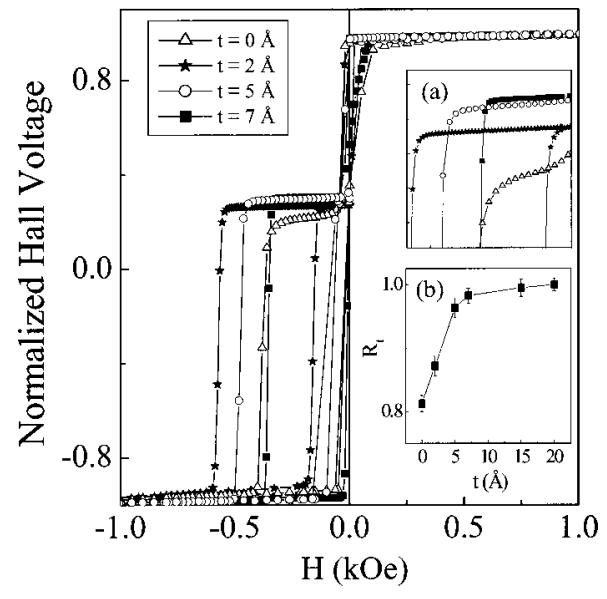

FIG. 3. (a) Normalized hysteresis loops corresponding to $[\mathrm{Pt}(20 \AA) / \mathrm{Co}(4 \AA)]_{3} / \mathrm{Pt}(20 \AA) / \mathrm{Cu}(30 \AA) /[\mathrm{Pt}(20 \AA) / \mathrm{Co}(4 \AA)]_{5} /$ $\mathrm{Pt}(t) / \mathrm{FeMn}(130 \AA)$, for $t=0,2,5$, and $7 \AA$. An enlargement of the central part of the hysteresis loops is shown in inset (a). Inset (b) gives the dependence of the magnetization amplitude ratio between the pinned and the free layers, $R_{t}$, on $t$.

The initial increase in the strength of FM-AFM exchange coupling as the Pt spacer layer thickness is varied can be qualitatively understood in terms of a better alignment of the last Co layer magnetization along the perpendicular-tofilm direction. This is well evidenced in Fig. 1(a), where, for $n=2$, the easy axis direction of the ML changes from being basically in-plane (for $t=0 \AA$ ) to perpendicular to plane (for $t=20 \AA$ ). For $n=5$, in spite of the square appearance of the hysteresis loops, it is reasonable to assume that some tilt in the Co magnetization could still be present, especially for the case where the $\mathrm{Co}$ is in direct contact with the AFM. In order to determine possible changes in the amplitude of the magnetization when $t$ is varied, a series of spin-valve-like samples with the composition $[\mathrm{Pt}(20 \AA) / \mathrm{Co}(4 \AA)]_{3} / \mathrm{Pt}(20 \AA) / \mathrm{Cu}(30 \AA) /[\mathrm{Pt}(20 \AA) /$ Co $(4 \AA)]_{5} / \mathrm{Pt}(t) / \mathrm{FeMn}(130 \AA)$ were prepared, the signal from the unpinned bottom multilayer being used as an internal calibration. The hysteresis loops for $t=0,2,5$, and $7 \AA$ are shown in Fig. 3. An enlargement of a part of these hysteresis loops [inset (a)] clearly shows that the magnetization of the pinned layer increases with $t$.

Quantification of this effect can be obtained by evaluating, for each value of $t$, the ratio between the magnetization amplitude (at the remanent state) of the pinned layer and that of the free layer, and normalizing to the ratio corresponding to $t=20 \AA$. This normalized ratio, $R_{t}$, is plotted in inset (b) of Fig. 3 as a function of $t$. It can be seen that the magnetization of the pinned multilayer increases by about $20 \%$ when going from $t=0 \AA$ to $t=20 \AA$.

Several authors have shown in the last years that exchange bias is roughly proportional to the FM-AFM spin projection at the interface. ${ }^{17}$ Their results indicate that $H_{E}$ is optimized when the FM and AFM easy axes are completely parallel to each other. In our case, during the perpendicular field cooling (FC) of as-deposited samples, the Co spins are basically aligned perpendicular to the film plane in the field of $2.5 \mathrm{kOe}$. As temperature is reduced to below the blocking temperature, the spins in the different FeMn crystallites orient along their easy axes closer to the FC direction. However, once at room temperature, when the field is removed, 
the Co spins, especially those of the last Co layer, tend to tilt at a certain angle towards in-plane, due to the induced easyplane anisotropy from the $\mathrm{Co} / \mathrm{FeMn}$ interface. This is clearly seen for $n=2$ in Fig. 1(a). Moreover, for $n=5$, one can see from Fig. 3(b) that if the angular tilt in the remanent state occurred only in the last Co layer, the magnetization of this layer should lie in-plane in order to account for the $20 \%$ loss of the magnetization amplitude. However, in this case, since the unidirectional direction was set perpendicular-to-plane, one would observe zero bias. Conversely, if all Co layers in the ML were so strongly coupled that the relative angular tilt between them was zero, the twist of the overall remanent magnetization would be of around $35^{\circ}$ for $t=0 \AA$. However, according to the difference in the magnetization amplitude, the relative tilt between magnetizations for $t=2 \AA$ and $t$ $=0^{\circ}$ would be of the order of $5^{\circ}$, which is too small to account for a difference in bias of a factor of 2 . Therefore, from these results one can infer that, for $n$ sufficiently large, a kind of partial domain wall is formed across the ML, the tilt of the Co magnetization increasing progressively when approaching the FM/AFM interface. The actual value of $H_{E}$ could then be related to the energy stored in these partial domain walls in the FM, similar to what has been suggested by some recent exchange bias models. ${ }^{18}$

Moreover, since FM-AFM exchange bias is mainly a short-range interaction, $H_{E}$ tends to exponentially decrease when increasing the nonmagnetic spacer layer thickness. ${ }^{4}$ Hence, the curves presented in Fig. 2 are the result of the interplay between two opposing effects: the increase of $H_{E}$ due to the role of the Pt spacer in reorienting the Co spins to make them lie more perpendicular to the film plane and its exponential decrease, typical of FM-nonmagnetic-AFM heterostructures. Note that the perpendicular anisotropy in $\mathrm{Pt} / \mathrm{Co}$ ML arises from hybridation effects between the Co and $\mathrm{Pt}$ wave functions at the Pt/Co interfaces. ${ }^{19-21}$ It should be noted that the angular tilt of Co spins is probably more pronounced for lower number of $\mathrm{Pt} / \mathrm{Co}$ repeats in the ML, where the perpendicular effective anisotropy is lower. This explains why the bias variation is markedly larger for lower values of $n$ (see Fig. 2).

Further evidence for the previous interpretation is obtained when another nonmagnetic (NM) spacer is used. If, at the FM-AFM interface, Pt is substituted by another metal which does not favor an effective perpendicular anisotropy in the ML, no $H_{E}$ enhancement is observed. This was confirmed using $\mathrm{Al}, \mathrm{Ru}$, and $\mathrm{Cu}$ as spacers in $[\mathrm{Pt}(20 \AA) / \mathrm{Co}(4 \AA)]_{5} / \mathrm{NM}(t) / \mathrm{FeMn}(130 \AA)$ samples. As can be seen in Fig. 4, $H_{E}$ decreases with $t$ in all these series.

Finally, one should not completely disregard that some other factors (e.g., structural ones) could also have some influence on the $H_{E}$ enhancement.

In conclusion, we have demonstrated that the presence of a nonmagnetic ultrathin layer between a FM and an AFM layer [e.g., a $\mathrm{Pt}$ spacer layer between a $(\mathrm{Pt} / \mathrm{Co}) \mathrm{ML}$ and FeMn] can enhance the magnitudes of $H_{E}$ and $H_{C}$. In this system, these enhancements are mainly governed by the role of the Pt/Co interface in increasing the net perpendicular magnetic moment of the last Co layer in the ML and the consequent FM-AFM spin projection at the interface.

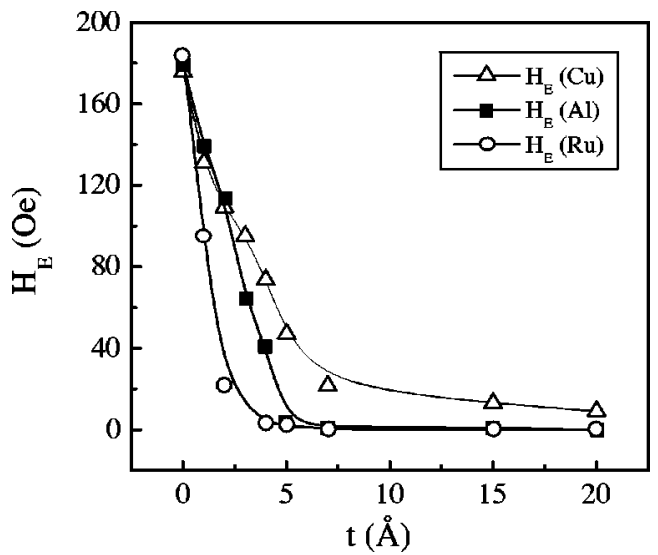

FIG. 4. Dependence of the exchange bias field, $H_{E}$, on the NM spacer thickness, $t$, in samples with the nominal composition [Pt $(20 \AA) / \mathrm{Co}(4 \AA)]_{5} / \mathrm{NM}(t) / \mathrm{FeMn}(130 \AA)$.

The authors thank I. Schuster and P. Bayle for their technical support and J. Nogues for the critical reading of the manuscript. F.G. acknowledges the CNPQ fellowship. This work was supported by the European Community through the NEXBIAS Grant No. HPRN-CT-2002-00296.

${ }^{1}$ B. Dieny, V. S. Speriosu, S. S. P. Parkin, B. A. Gurney, D. R. Wilhoit, and D. Mauri, Phys. Rev. B 43, 1297 (1991).

${ }^{2}$ J. C. S. Kools, IEEE Trans. Magn. 32, 3165 (1996).

${ }^{3}$ J. Nogués and I. K. Schuller, J. Magn. Magn. Mater. 192, 203 (1999).

${ }^{4}$ N. J. Gökemeijer, T. Ambrose, and C. L. Chien, Phys. Rev. Lett. 79, 4270 (1997). T. Mewes, B. F. P. Roos, S. O. Demokritov, and B. Hillebrands, J. Appl. Phys. 87, 5064 (2000); M.-T. Lin, C. H. Ho, C.-R. Chang, and Y. D. Yao, Phys. Rev. B 63, R100404 (2001).

${ }^{5}$ P. Miltényi, M. Gierlings, J. Keller, B. Beschoten, G. Güntherodt, U. Nowak, and K. D. Usadel, Phys. Rev. Lett. 84, 4224 (2000).

${ }^{6}$ T. Mewes, R. Lopusnik, J. Fassbender, B. Hillebrands, M. Jung, D. Engel,

A. Ehresmann, and H. Schmoranzer, Appl. Phys. Lett. 76, 1057 (2000)

${ }^{7}$ U. Nowak, K. D. Usadel, J. Keller, P. Miltényi, B. Beschoten, and G. Güntherodt, Phys. Rev. B 66, 014430 (2002).

${ }^{8}$ J.-V. Kim and R. L. Stamps, Appl. Phys. Lett. 79, 2785 (2001).

${ }^{9}$ B. Kagerer, Ch. Binek, and W. Kleemann, J. Magn. Magn. Mater. 217, 139 (2000).

${ }^{10}$ S. Maat, K. Takano, S. S. P. Parkin, and E. E. Fullerton, Phys. Rev. Lett. 87, 087202 (2001).

${ }^{11}$ F. Garcia, G. Casali, S. Auffret, B. Rodmacq, and B. Dieny, J. Appl. Phys. 91, 6905 (2002); F. Garcia, J. Moritz, F. Ernult, S. Auffret, B. Rodmacq, B. Dieny, J. Camarero, Y. Pennec, S. Pizzini, and J. Vogel, IEEE Trans. Magn. 38, 2730 (2002).

${ }^{12}$ M. T. Johnson, P. J. H. Bloemen, F. J. A. den Broeder, and J. J. de Vries, Rep. Prog. Phys. 59, 1409 (1996).

${ }^{13}$ O. Hellwig, S. Maat, J. B. Kortright, and E. E. Fullerton, Phys. Rev. B 65, 144418 (2002).

${ }^{14}$ N. Nishimura, T. Hirai, A. Koganei, T. Ikeda, and K. Okano, J. Appl. Phys. 91, 5246 (2002)

${ }^{15}$ F. Garcia, F. Fettar, S. Auffret, B. Rodmacq, and B. Dieny, J. Appl. Phys. 93, 8397 (2003).

${ }^{16}$ S. Zhang, Phys. Rev. B 51, 3632 (1995).

${ }^{17}$ J. Nogués, T. J. Moran, D. Lederman, I. K. Schuller, and K. V. Rao, Phys. Rev. B 59, 6984 (1999); C. Christides, N. Moutis, Ph. Komninou, Th. Kehagias, and G. Nouet, J. Appl. Phys. 92, 397 (2002).

${ }^{18}$ M. Kiwi, J. Mejía-López, R. D. Portugal, and M. Ramírez, Europhys. Lett. 48, 573 (1999).

${ }^{19}$ N. Nakajima, T. Koide, T. Shimada, H. Miyauchi, H. Fukutani, A. Fujimori, K. Iio, T. Katayama, M. Nývlt, and Y. Suzuki, Phys. Rev. Lett. 81, 5229 (1998)

${ }^{20}$ I. K. Schuller, S. Kim, and C. Leighton, J. Magn. Magn. Mater. 200, 571 (1999).

${ }^{21}$ M. T. Johnson, P. J. H. Bloemen, F. J. A. den Broeder, and J. J. de Vries, Rep. Prog. Phys. 59, 1409 (1996). 